\title{
Immunologic pericarditis following trauma to the pericardium
}

\author{
LBM Fernando ${ }^{1}$, Ajith Lamahewa ${ }^{2}$ \\ ${ }^{1}$ Consultant Judicial Medical Officer, General Hospital, Matara. \\ ${ }^{2}$ Senior Lecturer in Pathology, Faculty of Medicine, University of Ruhuna, Galle.
}

\section{Introduction}

Non-infectious acute pericarditis frequently complicates acute rheumatic fever, myocardial infarction, chronic renal failure and connective tissue disease.

Acute non-infectious pericarditis may occur following trauma to the pericardium. There is typically a delay of onset of pericarditis following trauma, supporting the aetio-pathogenesis concept of immunological hypersensitivity.

\section{Case History}

A 47 year old man was admitted to the General Hospital Matara with the history of stab injury to the right anterior chest. On admission the clinical picture was that of right haemothorax. Intercostal tube was inserted and blood drained out. Patient recovered and was discharged after 9 days. Two days later the patient was readmitted with breathlessness and chest pain. Clinical examination revealed tachycardia and hypotension and he died after 20 hours. Autopsy revealed the following findings.

The body was $160 \mathrm{~cm}$ tall and estimated to be 60 kg had healed sutured incised injury over the head and healed sutured stab injury over the right anterior chest. A surgical incision made for the insertion of intercostal tube was noticed over the right chest.

There was no evidence of head injury or brain damage. The pericardial sac was distended containing blood stained serous fluid. The pericardium appeared inflamed. The heart weighed $300 \mathrm{~g}$. The coronary arteries were free from atheroma. Myocardium and heart valves appeared normal.

An organised blood clot with $250 \mathrm{~mL}$ of fluid was found in the right pleural cavity. The stab injury was seen penetrating through the pleural cavity into the upper lobe of right lung at the level of $2^{\text {nd }}$ intercostals space. Length and depth of the lung injury measured $2.5 \mathrm{~cm}$ and $2 \mathrm{~cm}$ respectively.
Both lungs were heavy and congested. Rest of the organs was unremarkable.

Toxicological analysis performed at the Department of Government Analyst was negative. Histopathology examination of the heart revealed greatly thickened pericardium with mononuclear inflammatory cell infiltrate mainly comprising of lympho-plasmacytic cells with few macrophages. Pericardium also contained abundant fibrinous material with early granulation tissue formation. No evidence of suppuration, granuloma, or neoplasia is seen. The underlying myocardium appeared normal. The microscopic examination of the rest of the internal organs were unremarkable.

\section{Discussion}

Acute pericarditis caused by immunologic hypersensitivity is characterised by timed-lag of several days to few weeks between trauma and clinical onset and histological evidence of acute pericarditis with lympho-plasmacytic cell infiltration devoid of neutrophils. During the course of lag period immunologic reaction develops against new antigens exposed on the pericardium following trauma. In this case patient recovered almost completely from the injuries to the pleural cavity and the lung. Trauma to the pericardium was probably caused by the movements of illplaced intercostals tube but not by stab injury itself.

This view was supported by retrospective review of chest radiograph. Here we find an important medico-legal issue that death was not directly caused by stab injury but occurred as a consequence of stabbing.

\section{References}

1. Chandrasoma P, Clive R Taylor. Concise Pathology, $2^{\text {nd }}$ Edition.

2. Ramzi S Cotran, Vinay Kumar, Tucker Collins, Robin's Pathologic Basis of Diseases. $6^{\text {th }}$ Ed, Philadelphia, WB Saunders, 1999; 587-9. 